\title{
Mapa multimedia de bienes culturales y de la memoria de la península de Punta del Este
}

\author{
Multimedia map of cultural assets and the memory of the \\ peninsula of Punta del Este
}

\section{Gabriela Campodónico ${ }^{1}$, Facundo Bianchi², Nicolás García ${ }^{3}$, Camila Fernández ${ }^{4}$, Matías González ${ }^{5}$, Martín Fabreau ${ }^{6}$}

\begin{abstract}
El vínculo entre Turismo, Cultura, Patrimonio y Memoria es de larga data y descansa sobre una serie de dinámicas relativas a la construcción de identidades de los grupos humanos. Esta propuesta surge de la conjunción de intereses surgidos entre el ámbito académico y la sociedad civil local.

Desde la Licenciatura en Turismo radicada en el CURE-UdelaR, entendimos pertinente la creación de un espacio diverso e integrado en el que confluyan docentes, estudiantes y actores locales. Desde la sociedad civil local, las necesidades partieron a través de inquietudes y demandas relacionadas a la elaboración de productos turísticos vinculados al espacio urbano, el patrimonio y la memoria de Punta del Este.

Planteamos como objetivo general la elaboración de un mapa cultural y de la memoria. En este proceso, generamos insumos para contar en el futuro con un archivo de bienes patrimoniales materiales e inmateriales de la Península, al servicio de sus habitantes y visitantes.
\end{abstract}

Palabras clave: turismo, patrimonio, memoria

The link between Tourism, Culture, Heritage and Memory is of long standing and rests on a series of dynamics related to the construction of identities of human groups. The

\footnotetext{
${ }^{1}$ Directora Área de Estudios Turísticos, FHCE -CURE. gcampod@yahoo.com

${ }^{2}$ Facundo Bianchi - Docente Ayudante Área de Estudios Turísticos, FHCE-CURE. facundo.bianchi@cure.edu.uy

${ }^{3}$ Nicolás García - Estudiante Licenciatura en Turismo, CURE. nicolas.garcia.pad@gmail.com

${ }^{4}$ Camila Fernández - Licenciada en Turismo, CURE. camilafernandez055@gmail.com

${ }^{5}$ Matías González - Estudiante Licenciatura en Turismo, CURE. matig5023@hotmail.com

${ }^{6}$ Martín Fabreau - Docente Adjunto Área de Estudios Turísticos, FHCE-CURE. fabreau@gmail.com
} 
present proposal arises from the combination of interests arising between the academic sphere and local civil society.

From the Degree in Tourism based in the CURE-UDELAR, we understood the creation of a diverse and integrated space in which teachers, students and local actors converge. From the local civil society, the needs started through concerns and demands related to the development of tourism products linked to the urban space, heritage and memory of Punta del Este.

We propose as a general objective the elaboration of a cultural and memory map. In this process, we generate inputs to count in the future with a file of material and immaterial heritage assets of the Peninsula, at the service of its inhabitants and visitors.

Keywords: tourism, heritage, memory 


\section{INTRODUCCIÓN}

Este trabajo surge de la conjunción de intereses y demandas entre el ámbito académico y la sociedad local. El punto de partida fue una serie de inquietudes y demandas relativas a la elaboración de productos turísticos concretos vinculados al espacio urbano, el patrimonio y la memoria de Punta del Este. La Comisión de Cultura y Patrimonio de la Alcaldía de Punta del Este requirió la colaboración de la Universidad para trabajar en el registro de bienes patrimoniales de la Península. Desde la Licenciatura en Turismo que se dicta en el CURE-UdelaR, entendimos necesario y pertinente la creación de un espacio/contexto diverso e integrado en el que participen docentes, estudiantes, egresados y actores locales y en donde actividades de Investigación, Extensión y Docencia tuvieran lugar. Paralelamente, también hay un vínculo construido y consolidado tanto con la Intendencia Municipal de Maldonado como con la Liga de Fomento y Turismo de Punta del Este, quienes por su parte tienen una estrecha relación con la Alcaldía de Punta del Este. Entendemos que esta convergencia entre la UdelaR y la Sociedad Civil hace propicio el comienzo de un espacio de trabajo en conjunto que tendrá como cometido la realización de variadas actividades de las que diversos actores sean partícipes y en donde se interrelacionan investigación, enseñanza y extensión (Campodónico y Fabreau, 2017).

\section{OBJETIVOS}

Este emprendimiento tiene como objetivo general elaborar un mapa multimedia cultural y de la memoria, donde se haga presente el vasto sistema de bienes culturales y patrimoniales, tangibles e intangibles, materiales y/ o materializables que configuran las diversas formas de representar, evocar y apropiar el espacio en general y el espacio urbano en particular de la Península de Punta del Este.

A lo anterior, se suman algunos objetivos específicos. En primer lugar, elaborar un mapa multimedia en donde figuren y se relacionen bienes patrimoniales y culturales emblemáticos de la península integrando diferentes tipos de registro (escrito, sonoro, visual, audiovisual). Luego, contribuir al desarrollo del turismo cultural en Punta del Este. También contribuir a la preservación de la memoria colectiva de los habitantes de Punta del Este, así como al fortalecimiento de su patrimonio material e inmaterial. Por último, iniciar la elaboración de un archivo de bienes patrimoniales materiales e inmateriales de la península, al servicio de sus habitantes y visitantes, que a su vez sirva de insumo para la elaboración de productos turísticos concretos.

\section{ESTRATEGIAS DE INVESTIGACIÓN}

El eje que orienta las actividades que vienen siendo desarrolladas tiene que ver con la identificación y caracterización de bienes culturales, bienes patrimoniales y lugares de memoria tanto para la salvaguarda patrimonial y de la memoria local, como también para elaboración de itinerarios urbanos con fines turísticos. De lo anterior se desprende que además de la elaboración de un mapa de bienes culturales y de la memoria, este proceso pauta además la elaboración de un registro/archivo en donde se identifican y caracterizan diversos bienes culturales tangibles e intangibles (Campodónico y Fabreau, 2017). 
Las actividades de investigación están pautadas por la utilización de una metodología cualitativa proveniente de la tradición antropológica en general y etnográfica en particular, en interfase con el trabajo de investigación en Turismo. Por otro lado, se integran también instancias participativas.

Concretamente, las prácticas de investigación comprenden trabajo de gabinete, trabajo de campo e instancias participativas junto a la sociedad civil.

En lo que hace a las actividades de gabinete hasta el momento se ha realizado trabajo de archivo, así como búsqueda bibliográfica y análisis de diversos tipos de documentos. El trabajo de campo se viene desarrollando tanto en Punta del Este co mo en Maldonado y también en Montevideo. Básicamente consiste en la realización de observaciones, entrevistas en profundidad y registro audiovisual.

A lo anterior se le agrega tanto investigación como planificación de las actividades; básicamente suelen realizarse reuniones periódicas con representantes de la Alcaldía de Punta del Este con quienes estamos en estrecha colaboración.

\section{AVANCES DE INVESTIGACIÓN}

Hasta el momento y desde el año 2016 este grupo de trabajo ha desarrollado algunas actividades que engloban la investigación, la docencia y la extensión, muchas de ellas planificadas en acuerdo con la Comisión de Patrimonio de la Alcaldía de Punta del Este.

En primer lugar, se realizó una jornada de presentación en el Espacio ANCAP - Alcaldía de Punta del Este a mediados de 2016, de trabajos estudiantiles de la Licenciatura en Turismo, producidos en el marco del curso Antropología del Turismo, sobre la caracterización de algunos lugares turísticos y bienes patrimoniales locales.

Paralelamente a ello, el equipo de trabajo ha realizado hasta el momento un total de doce entrevistas en profundidad de carácter exploratorio a diversos informantes calificados. Del procesamiento y sistematización de la información recolectada se generó una lista de categorías descriptivas (lugares, personas, acontecimientos, saberes/ oficios y prácticas más relevantes), sintetizadas en un documento unificado. Dicho documento le fue facilitado a los entrevistados con el fin de ratificar o rectificar dicho listado. Luego de procesar esas devoluciones saldrá un listado definitivo con el que se trabajará en la fase siguiente.

En este proceso de entrevistas/devolución, se ha generado una base de datos con posibles personas a entrevistar, a partir de los aportes de cada entrevistado actual, que refieren a determinados actores relevantes en la historia de la península.

Paralelamente a la realización de entrevistas, en el marco de los festejos por los ciento diez años del balneario, el 26 de julio de 2016, se desarrolló el evento "Panel de la Memoria" en el salón de la Liga de Fomento de Punta del Este; actividad gestionada por el equipo de trabajo y representantes de la Comisión de Cultura y Patrimonio del Municipio de Punta del Este. Los panelistas participantes fueron Mecha Gattás, Yolanda Clavijo y Luis Sáder.

Además de ser un ejercicio de memoria colectiva, de evocación y de objetivación, la jornada tuvo entre sus principales objetivos transmitirle a la comunidad el trabajo que se viene realizando, así como también la generación de un intercambio entre los panelistas y el público. A su vez, la generación de nuevos contenidos basados en los relatos esbozados por los panelistas invitados.

En lo que hace a actividades de educación, entre los meses de agosto y octubre, se dictó el curso de Educación Permanente "Memoria y Patrimonio desde la Antropología del Turismo". Realizado en el CURE por los docentes Gabriela Campodónico y Martín 
Fabreau con una carga horaria de treinta horas; el mismo estuvo dirigido a egresados de la Licenciatura en Turismo y de carreras afines, profesionales, estudiantes avanzados de turismo y actores de la sociedad civil interesados en el tema. Es así, que, contribuyendo a la consolidación del vínculo con la sociedad civil, parte de los integrantes de la Comisión de Patrimonio y Cultura del Municipio de Punta del Este, participan en la realización del curso.

También se realizaron actividades junto a la Escuela $N^{\circ} 5$ de Punta del Este en el contexto del Día Mundial del Turismo. Concretamente se trabajó en la elaboración y puesta en práctica de un circuito turístico junto a estudiantes y docentes de la institución.

\section{PERSPECTIVAS}

Al día de hoy, el equipo se encuentra sistematizando las devoluciones realizadas por los entrevistados sobre las categorías emergidas de la fase exploratoria. La idea será generar una lista definitiva en donde se identifique los bienes culturales entendidos como relevantes. A su vez, lo anterior se triangulará con datos bibliográficos, realización de nuevas entrevistas e instancias participativas.

El listado de categorías emergidas será trabajado de manera exhaustiva en la próxima etapa del trabajo.

A todo esto, parte de la información sistematizada hasta el momento viene siendo plasmada en un mapa; al día de hoy se cuenta con una identificación y ubicación de un gran número de lugares y bienes culturales, algunos de ellos aún existentes y otros presentes en la memoria de los habitantes locales.

Con miras a futuro, se comenzará a trabajar en una caracterización exhaustiva de cada bien cultural, así como en la continuación de su mapeo.

Al haber trabajado principalmente con informantes calificados, en su mayoría de sectores medios, hemos ido viendo la necesidad de incluir en próximas etapas, entrevistados pertenecientes a sectores populares con miras a ampliar tanto el listado de bienes como las formas de apropiación de estos.

Una vez que se haya arribado a una caracterización exhaustiva y sistemática de los bienes culturales identificados, se procederá a la elaboración del producto final: el mapa multimedia propiamente dicho.

La idea es generar un soporte georreferenciado para Punta del Este en donde se puedan combinar registros escritos, visuales y sonoros y en donde se vean representados bienes culturales materiales e inmateriales, así como lugares de memoria, existentes o que ya no están presentes.

\section{BIBLIOGRAFÍA}

Augé, M. (1992). Los No Lugares. Espacios Del Anonimato. Una Antropología de la Sobremodernidad. Barcelona: Ed. Gedisa S.A.

Augé, M. (2007). Por una Antropología de la Movilidad. Barcelona: Gedisa.

Campodónico, G., Fabreau, M. (2017). Mapa multimedia de bienes culturales y de la memoria. Proyecto presentado Ilamado VUSP-CSIC.

Candau, J. (2005). Antropologia da Memória. Instituto Piaget. Lisboa. 
CSEAM. Presentación - "Visión y Misión" En: http:// www.extension.udelar.edu.uy/ mision-y-vision/

Fabreau, M. (2014). Entre Múltiples Alteridades Móviles. Sobre el Conocimiento Antropológico y Algunas Interfases con los Estudios Turísticos.

Falero, A. y Campodónico, R. (comp.) El Turismo Bajo la Lupa Académica, Biblioteca Plural, CSIC. 21-40. Montevideo.

Guber, R. (2004). El salvaje metropolitano. Reconstrucción del conocimiento social en el trabajo de campo. Buenos Aires: Editorial Paidós.

Halbwachs, M. (1950). La Memoria Colectiva. Prensa Universitaria de Zaragoza. España.

Ministerio de Turismo (2016). Anuario 2016. Estadísticas del Turismo. Turismo receptivo. Anuario 2015. Estadísticas del Turismo. Turismo receptivo. En: www.mintur.gub.uy.

Pascale, R.; Varela, L. (2011). Resultados de investigación para Proyecto IDEAR, Maldonado, Uruguay. versión pdf.

Prats, LI. (1997). Antropología y Patrimonio. Barcelona: Ariel.

Rosas Mantecón, A. (2005). Las disputas por el patrimonio. Transformaciones analíticas y contextuales de la problemática patrimonial en México En: García Canclini, N. (coord.), La antropología urbana en México, (pp 60-95) México D.F., México: Fondo de Cultura Económica, Consejo Nacional para la Cultura y las Artes y Universidad Autónoma Metropolitana.

Santana, A. (1997). Antropología y turismo. ¿Nuevas hordas, viejas culturas? Barcelona: Ariel-Antropología.

Santana, A. (2003). Turismo cultural, culturas turísticas. Horizontes Antropológicos (Porto Alegre), 9, 20.

Scagliola, A., Rodríguez, M. (coords.). (2013). Agenda Estratégica. Plan de desarrollo social departamental. Maldonado 2013 - 2015. Uruguay Social, Presidencia de la República.

Veiga, D. (coord.). (2011). Crecimiento económico y desigualdad social en Maldonado. Informe del Estudio realizado en el marco del Convenio entre la Intendencia de Maldonado, la Facultad de Ciencias Sociales de la Universidad de la República y la Asociación Pro-fundación para las Ciencias Sociales. FCS, CURE-UDELAR, Maldonado. 